\title{
Metastasis of renal cell carcinoma around suture anchor implants
}

\author{
Samuel Baek ${ }^{1}$, Myung Ho Shin ${ }^{2}$, Tae Min Kim³ ${ }^{3}$ Kyung-Soo Oh ${ }^{4}$, Dong Ryun Lee ${ }^{4}$, Seok Won Chung ${ }^{4}$ \\ ${ }^{1}$ Department of Orthopedic Surgery, Seoul Red Cross Hospital, Seoul, Korea \\ ${ }^{2}$ Department of Orthopedic Surgery, CM Hospital, Seoul, Korea \\ ${ }^{3}$ Department of Orthopedic Surgery, Yonsei Gunwoo Hospital, Seoul, Korea \\ ${ }^{4}$ Department of Orthopedic Surgery, Konkuk University School of Medicine, Seoul, Korea
}

We present an unusual case of bone metastases from renal cell carcinoma around orthopedic implants in a 78-year-old female with osteolytic, expansile, highly vascularized, malignant infiltration around suture anchors in the proximal humerus. The patient had undergone arthroscopic rotator cuff repair using suture anchor implants 6 years previously. After diagnosis of bone metastasis, she was successfully treated with metastasectomy and internal fixation using a plate and screws, with cement augmentation. This report is the first to document metastases around a suture anchor in a bone and suggests the vulnerability of suture anchor implants to tumor metastasis.

Keywords: Metastasis; Renal cell carcinoma; Suture anchor; Orthopedic implant; Metastasectomy

Renal cell carcinoma (RCC) originates from the epithelium of the proximal convoluted renal tubules. RCC has a propensity for metastasis, and the frequency of spread varies: $60 \%-75 \%$ spread to the lungs, $60 \%-65 \%$ to the regional lymph nodes, $39 \%-40 \%$ to the liver, $20 \%-35 \%$ to the bones, and $5 \%-7 \%$ to the brain [1]. Even though more than $60 \%$ of RCC patients develop various metastases during the course of their disease, not many reports describe metastasis around orthopedic implants. Here, we present a case of RCC that metastasized to the proximal humerus in a patient who had undergone repair of a torn rotator cuff using suture anchors 6 years prior. She was successfully treated with metastasectomy with cementation and internal fixation. We discuss the diagnostic and therapeutic perspectives and briefly review the relevant literature.

\section{CASE REPORT}

The approval of the Institutional Review Board of Konkuk University Medical Center (IRB No. 2020-09-025) was obtained and, informed consent for this retrospective study was waived by the Board.

A 78-year-old female was referred for pain and pseudoparesis of the right shoulder along with a firm, bulging mass. Six years previous, she had undergone arthroscopic rotator cuff repair (RCR) for a painful and large rotator cuff tear using crossFT (Conmed, Utica, NY, USA) suture anchors at the medial footprint and SwiveLock SP (Arthrex, Naples, FL, USA) anchors at the lateral humeral cortex. The crossFT anchor was composed of polyether ether ketone materials, and the SwiveLock SP anchor had a titanium tip. The patient's symptoms improved after RCR,

Received: March 24, $2021 \quad$ Revised: April 25, $2001 \quad$ Accepted: April 28, 2021

Correspondence to: Seok Won Chung

Department of Orthopedic Surgery, Center for Shoulder and Elbow Surgery, Konkuk University School of Medicine, 120 Neungdong-ro, Gwangjin-gu, Seoul 05029, Korea

Tel: +82-2-2030-7604, Fax: +82-2-2030-7748, E-mail: smilecsw@gmail.com, ORCID: https://orcid.org/0000-0002-8221-9289

\section{Financial support: None.}

Conflict of interest: None.

CopyrightC 2021 Korean Shoulder and Elbow Society.

This is an Open Access article distributed under the terms of the Creative Commons Attribution Non-Commercial License (http://creativecommons.org/licenses/by-nc/4.0/) which permits unrestricted non-commercial use, distribution, and reproduction in any medium, provided the original work is properly cited. 
and postoperative images showed well-maintained continuity and thickness of the repaired tendon. Two weeks after RCR, a 7.9-cm-sized heterogeneous mass was detected in the upper pole of the left kidney, and she underwent laparoscopic nephrectomy one week later. The final diagnosis was grade 3 clear cell RCC.

About 75 months after RCR, a simple X-ray showed an expansile lytic bone lesion with an ill-defined border and a broad zone of transition in the right proximal humerus around anchor implants (Fig. 1A and B). Magnetic resonance imaging showed a T2-hyperintense and T1-hypointense, $4.3 \times 3.5 \times 4.3$-cm-sized, poorly circumscribed, cortical-destructive lesion with peri-osseous mass formation (Fig. 1C and D). The findings suggested metastasis, and infection or anchor foreign body reactions were unlikely. Collaborating with colleagues in the Departments of Medical Oncology and Radiation Oncology, we planned the patient's postoperative treatment, such as palliative radiotherapy or systemic chemotherapy. A whole-body bone scan to identify additional metastatic lesions prior to surgery demonstrated foci, suggesting the possibility of bone metastases in the left ischium, lateral condyle of the left femur, and distal shaft of the right femur in addition to the primary focus (Fig. 1E). Because the patient did not report additional symptoms that correlated with the scan result, further evaluation was not performed.

For treatment of this metastatic lesion, she underwent metastasectomy without preoperative embolization and internal fixation with cement augmentation. During the metastasectomy, an exophytic highly vascular mass with several feeding arteries surrounded by lateral anchors was found and removed. After surgery, only a friable medial cortex remained, and the distal humerus was impacted into the humeral head. Therefore, plate (PHILOS; DePuy Synthes, Raynham, MA, USA) fixation with cement augmentation was performed to increase stability (Fig. 2A-D). Histopathology confirmed that the lesion was metastatic clear cell RCC (Fig. 3).

The patient's postoperative course was uneventful. One month after surgery, she started Votrient (pazopanib) chemotherapy, resulting in symptomatic improvement in her right shoulder. Two months after surgery, she could dress herself, and the range of motion improved, with $70^{\circ}$ of active elevation, active deltoid muscle contracture. A postoperative chest radiograph demon-
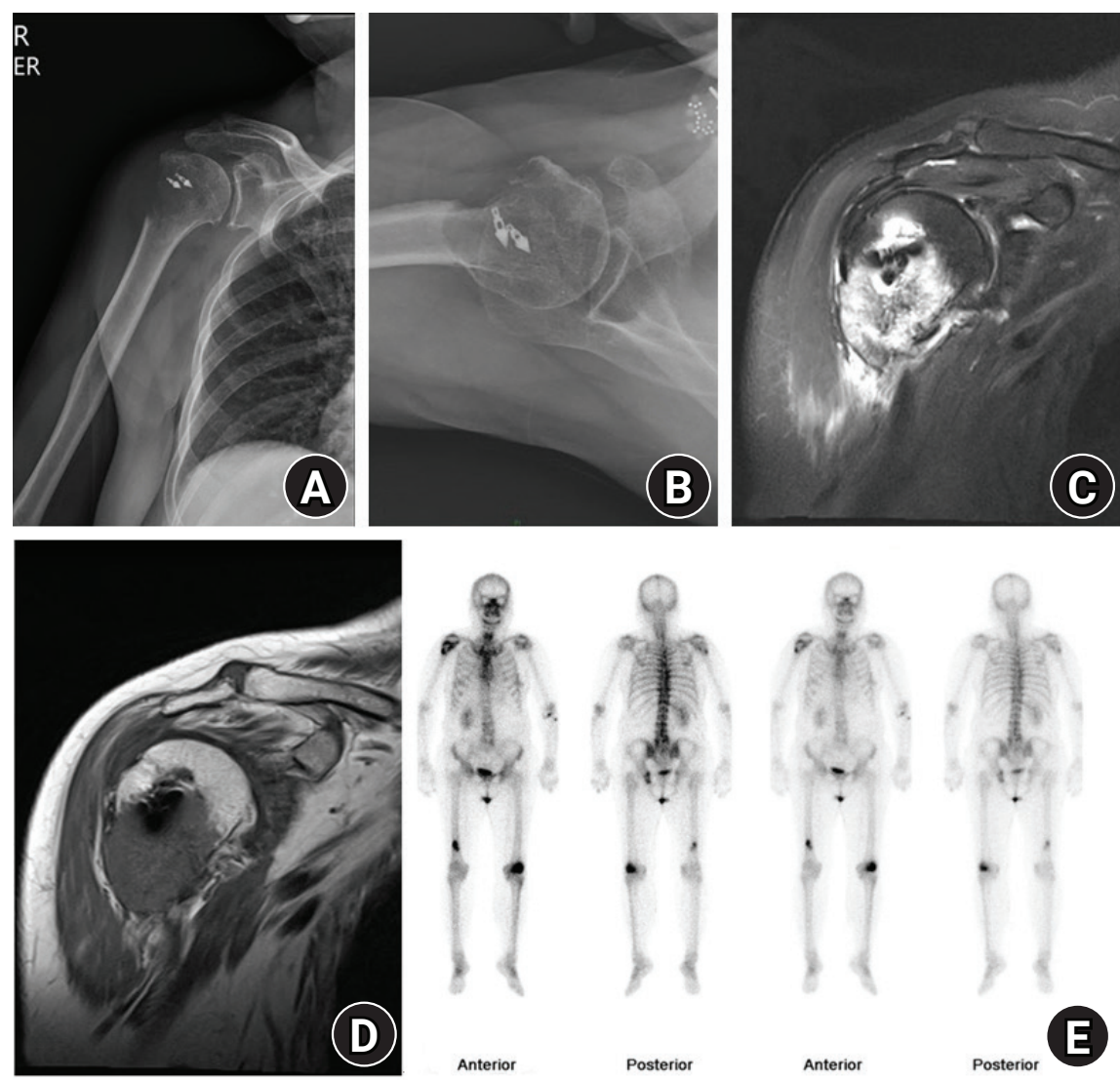

Fig. 1. Anteroposterior (A) and axial (B) radiographs demonstrated an osteolytic radiolucent lesion of the proximal humerus that also surrounded suture anchor implants. T2 coronal (C) and T1 coronal (D) magnetic resonance images revealed a cortical destructive mass lesion with peri-osseous mass formation. (E) A whole-body bone scan demonstrated multiple bone metastases. 

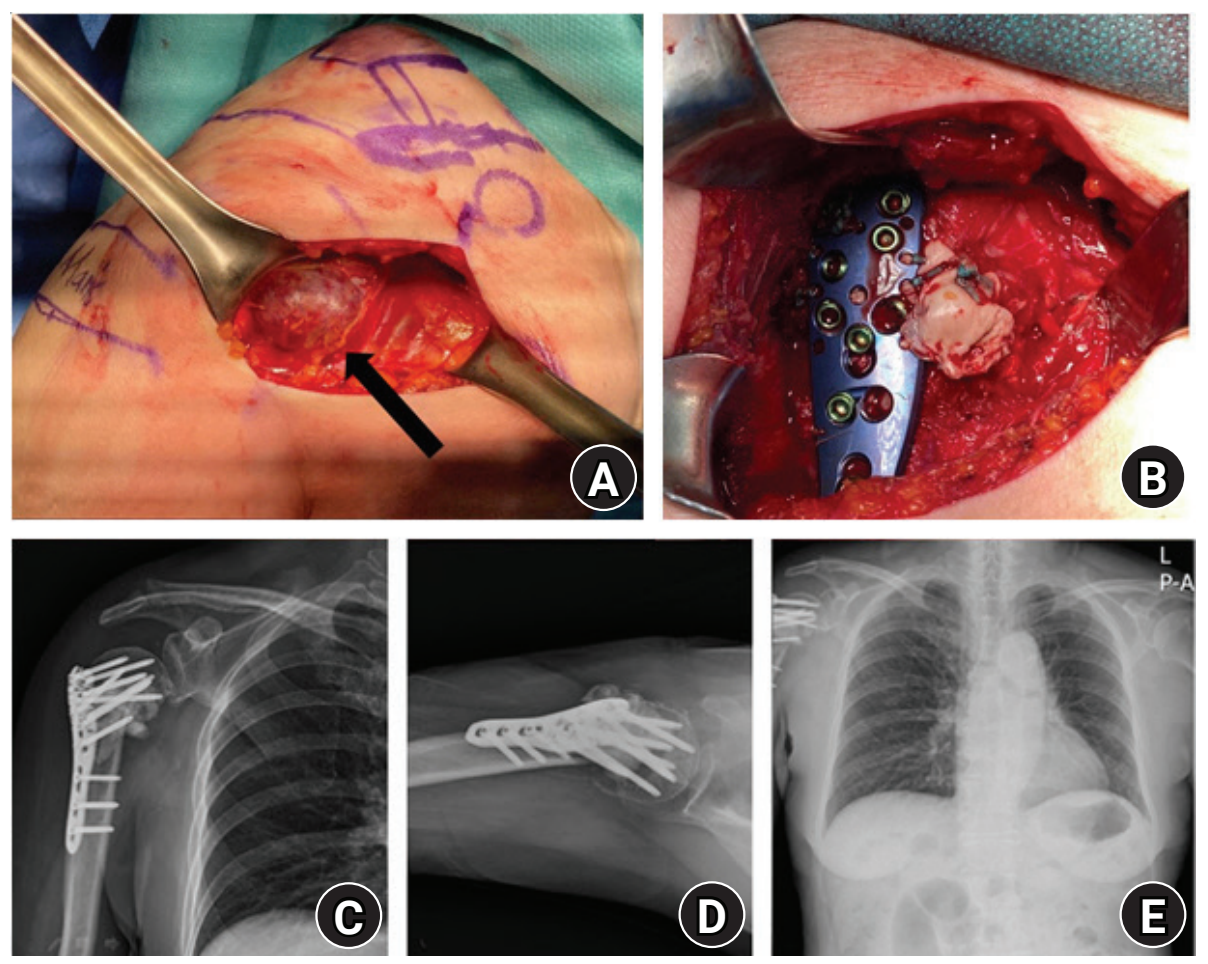

Fig. 2. An expansile metastatic mass (black arrow) (A) was observed intraoperatively, followed by plate fixation and cement augmentation (B). Immediate postoperative anteroposterior (C) and axial (D) radiographs demonstrated the metastasectomy margin, and postoperative chest radiograph (E) revealed neither fixation loss nor recurrence at 8 months following surgery.

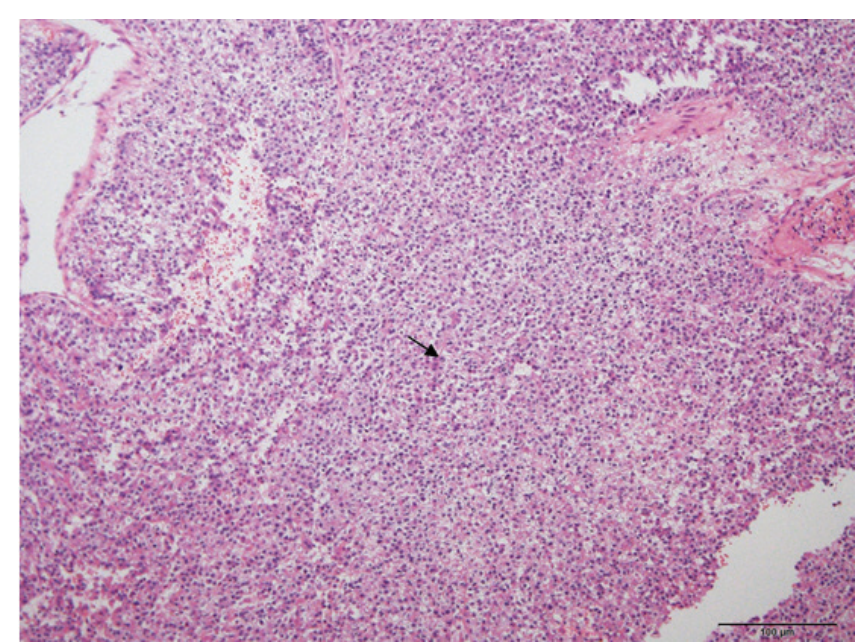

Fig. 3. High-power photomicrography $(\mathrm{H} \& \mathrm{E}, \times 100)$ confirmed metastatic renal cell carcinoma with polyclonal cells containing abundant clear cytoplasm and round-to-oval hyperchromatic nuclei, similar to clear cell change (black arrow).

strated no evidence of fixation loss or recurrence (Fig. 2E).

\section{DISCUSSION}

RCC is classified into clear cell, papillary, chromophobe, multilocular cystic, collecting duct, medullary, and unclassified types according to histology. The clear cell type is most frequently reported. Bone metastasis, observed in one-third of patients with RCC, has a higher incidence in patients with clear cell type RCC [1]. Resection of the metastases followed by local and systemic therapies is reported to improve prognosis. Surgical excision, however, can be difficult because metastases tend to be large, progressive, highly destructive, hypervascularized, and associated with a soft-tissue mass.

Only a few studies have previously reported metastasis around orthopedic implants, and most of these reports were confined to implants of total arthroplasty or plates [2,3]. The reported cases of metastasis around orthopedic implants have originated from non-Hodgkin's lymphoma, malignant fibrous histiocytoma, immunoblastic lymphoma, carcinomas of the lung, gastric carcinoma, endometrial carcinoma, ovarian carcinoma, RCC, hepatocellular carcinoma, and prostate cancer [2-4]. Although various cancers have been reported, no definite common denominator exists among cases of metastasis. Most metastatic lesions, however, showed periprosthetic osteolysis around the metal implants. Although we do not know the exact mechanism of metastasis around implants (or whether there is a cause-and-effect relationship), we believe that dissemination of cancer cells via blood vessels to tissues weakened by surgical trauma can affect the course 
of metastasis, and more cancer cells can accumulate on implants, which are foreign bodies [5].

Regarding RCC metastases to orthopedic implants, three case reports described metastases around total hip arthroplasty implants [6-8]. Two occurred around femoral implants [6,8], and one occurred around an acetabular implant [7]. All three cases showed loosening of the implants by metastatic infiltration. Two cases $[7,8]$ were treated by revision arthroplasty, and one case [6] utilized open biopsy of the femoral bone for diagnosis followed by palliative treatment.

No previous reports documented metastasis around a suture anchor inserted in bone. RCC is a highly vascularized tumor with extensive, thin-walled, sinusoid-like vessels, producing areas with a rich blood supply (such as the vertebrate or pelvis) prone to metastasis. We believe this feature of blood-born RCC metastasis can cause cancer cells to spread around the suture anchor at a site of surgical trauma. Several authors have postulated that abnormal blood flow increases due to surgical insult and the healing response, and this increased blood flow can create a predilection for metastases. In addition, a hematoma formed during RCR could be a hotbed for cancer cell seeding [9]. Furthermore, the suture anchor implant is a foreign body inside a bone, which can cause a foreign body reaction, such as activation of macrophages, giant cells, and leukocytes [10]. This foreign body reaction can facilitate the growth of blood-borne tumors, as suggested by Murphy et al. [5] However, these explanations for increased susceptibility to metastasis around implants are only hypotheses and are not fully understood; thus, they warrant further study. We believe our case demonstrates the vulnerability of suture anchor implant after RCR to increase susceptibility to tumor metastasis.

This is the first report documenting a case of RCC metastasis around a suture anchor implant after RCR. Clinicians must maintain a high index of suspicion and consider the possibility of metastases around orthopedic implants in patients with malignancies of various origins.

\section{ORCID}

Samuel Baek

https://orcid.org/0000-0001-9904-1184

Myung Ho Shin

https://orcid.org/0000-0002-2134-6946
Tae Min Kim

Kyung-Soo Oh

Dong Ryun Lee

Seok Won Chung

https://orcid.org/0000-0002-3977-536X

https://orcid.org/0000-0002-9812-9130

https://orcid.org/0000-0001-5305-1475

https://orcid.org/0000-0002-8221-9289

\section{REFERENCES}

1. Khan S, Awan SA, Jahangir S, Kamran S, Ahmad IN. Bone marrow metastasis in clear cell renal cell carcinoma: a case study. Cureus 2019;11:e4181.

2. Troop JK, Mallory TH, Fisher DA, Vaughn BK. Malignant fibrous histiocytoma after total hip arthroplasty: a case report. Clin Orthop Relat Res 1990;(253):297-300.

3. Bali K, Prabhakar S, Krishnan V, Dhillon MS. Hip hemiarthroplasty periprosthetic loosening caused by papillary ovarian carcinoma metastasis in a 78-year-old woman: a rare presentation and a literature review. Am J Orthop (Belle Mead NJ) 2012;41: 33-6.

4. Nagy MT, Young AF, Gorgees N, Barrington RL. Periprosthetic metastasis: a rare cause for prosthetic loosening. BMJ Case Rep 2013;2013:bcr2013008707.

5. Murphy P, Alexander P, Senior PV, Fleming J, Kirkham N, Taylor I. Mechanisms of organ selective tumour growth by bloodborne cancer cells. Br J Cancer 1988;57:19-31.

6. Dramis A, Desai AS, Board TN, Hekal WE, Panezai JR. Periprosthetic osteolysis due to metastatic renal cell carcinoma: a case report. Cases J 2008;1:297.

7. Simon JP, Bellemans J, Samson I. Metastasis from renal cell carcinoma presenting as osteolysis in total hip arthroplasty: a case report. Acta Orthop Belg 2008;74:122-4.

8. Bonnevialle P, Brouchet A, Sans N, Chevreau C, Roche H. Metastasis on previous hip arthroplasty: three cases. Rev Chir Orthop Reparatrice Appar Mot 2005;91:558-63.

9. Dib LL, Soares AL, Sandoval RL, Nannmark U. Breast metastasis around dental implants: a case report. Clin Implant Dent Relat Res 2007;9:112-5.

10. Kim JH, Kim JI, Lee HJ, et al. Long-term follow-up of extensive peri-anchor (Poly-L/D-lactic Acid) cyst formation after arthroscopic rotator cuff repair: a case report. Clin Shoulder Elbow 2019;22:100-5. 